\title{
Myotonic dystrophy diagnosed during the perinatal period: A case series report
}

\author{
You Jung Shin', Do Jin Kim², So Yeon Park², Jin Hoon Chung ${ }^{3}$, Yeon Kyung Lee ${ }^{4}$, and Hyun Mee Ryu ${ }^{2,3, *}$ \\ ${ }^{1}$ Department of Obstetrics and Gynaecology, Hankook General Hospital, Jeju, Korea \\ ${ }^{2}$ Laboratory of Medical Genetics, Cheil General Hospital and Women's Healthcare Center, Dankook University College of Medicine, Seoul, Korea \\ ${ }^{3}$ Division of Maternal-Fetal Medicine, Department of Obstetrics and Gynaecology, Cheil General Hospital and Women's Healthcare Center, \\ Dankook University College of Medicine, Seoul, Korea \\ ${ }^{4}$ Department of Pediatrics, Cheil General Hospital and Women's Healthcare Center, Dankook University College of Medicine, Seoul, Korea
}

Congenital myotonic dystrophy (CMD) which is transmitted in an autosomal-dominant manner, can also be observed in newborns born to asymptomatic parents who have a myotonic dystrophy type 1 or premutation allele, especially from the mother. A mother with myotonic dystrophy could be subfertile and the pregnancy could be complicated with the risk of a preterm birth. Newborns with CMD may demonstrate symptoms such as hypotonia and poor motor activity, as well as respiratory and feeding difficulties. Additionally, CMD has a high mortality rate at birth. Detection of the signs and symptoms during pregnancy is helpful for a prenatal diagnosis of $\mathrm{CMD}$ in cases where the family history is not known.

Key words: Myotonic dystrophy, Congenital, Pregnancy, Genetic counseling, Postpartum period.

\section{Introduction}

Myotonic dystrophy type 1 (DM1, Curschmann-Steinert disease, OMIM 160900) is a rare disorder, but remains the most common adult muscular dystrophy. DM1 is characterized by autosomal dominant inheritance and multisystemic symptoms, including myotonia, muscle wasting, cardiac conduction defects, insulin resistance, cataracts, and cognitive dysfunction. DM 1 is caused by the expansion of an unstable cystosinethymine-guanine repeat located in the 3'-untranslated region of the myotonic dystrophy (DM) protein kinase gene on chromosome 19q13.3 [1,2]. The pregnancy and postpartum periods in a mother with classic DM are usually complicated and there is a possibility of conceiving a child with congenital myotonic dystrophy (CMD); however, the disorder often has not been diagnosed beforehand. Although DM is a systemic disease for which there is no complete cure, early diagnosis remains important as effective management may reduce the morbidity and mortality of patients and help them to plan their pregnancies. Here, we report four cases in which the prenatal and postnatal data were available from March 2009 to December 2010 at Cheil General Hospital.

\section{Case}

\section{Case 1}

A 31-year old Korean woman, II-2 (Fig. 1A), gravida 3 para 1, living baby 0 was referred to our institution with infertility of

Received: 25 October 2016, Revised: 12 December 2016, Accepted: 12 December 2016, Published: 31 December 2016

*Corresponding author: Hyun Mee Ryu, M.D., Ph.D.

Division of Maternal-Fetal Medicine, Department of Obstetrics and Gynaecology, Laboratory of Medical Genetics, Cheil General Hospital and Women's Healthcare Center, Dankook University College of Medicine, 17 Seoae-ro 1-gil, Jung-gu, Seoul 04619, Korea.

Tel: +82-2-2000-7683, Fax: +82-2-2278-4574, E-mail: hmryu2012@naver.com

Conflict of interest: The authors declare that they do not have any conflicts of interest.

(c) This is an open-access article distributed under the terms of the Creative Commons Attribution Non-Commercial License (http://creativecommons.org/licenses/by-nc/4.0/) which permits unrestricted non-commercial use, distribution, and reproduction in any medium, provided the original work is properly cited.

(c) Copyright 2016 by the Korean Society of Medical Genetics

www.e-kjgm.org 
A

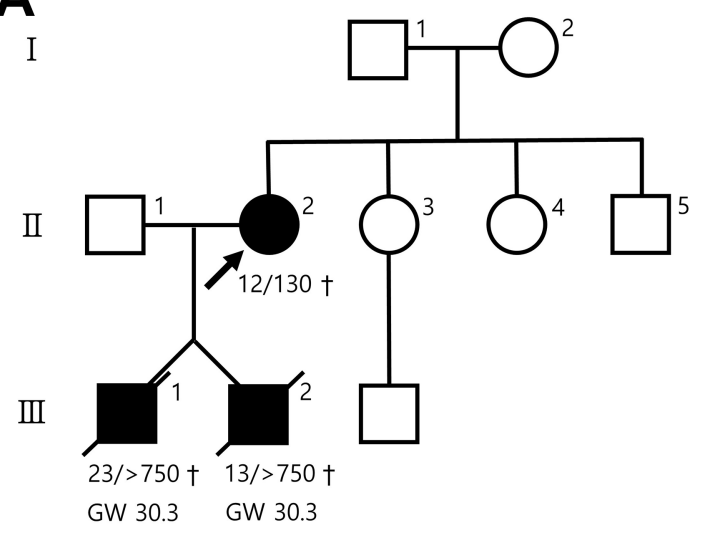

C

I

II

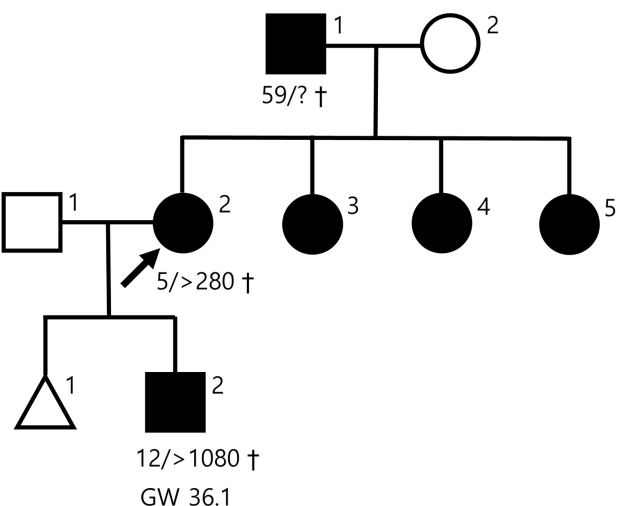

B

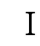

II

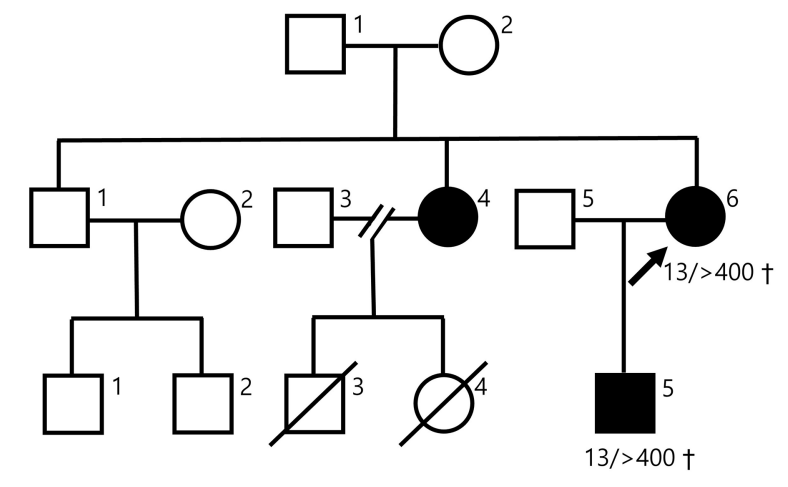

D

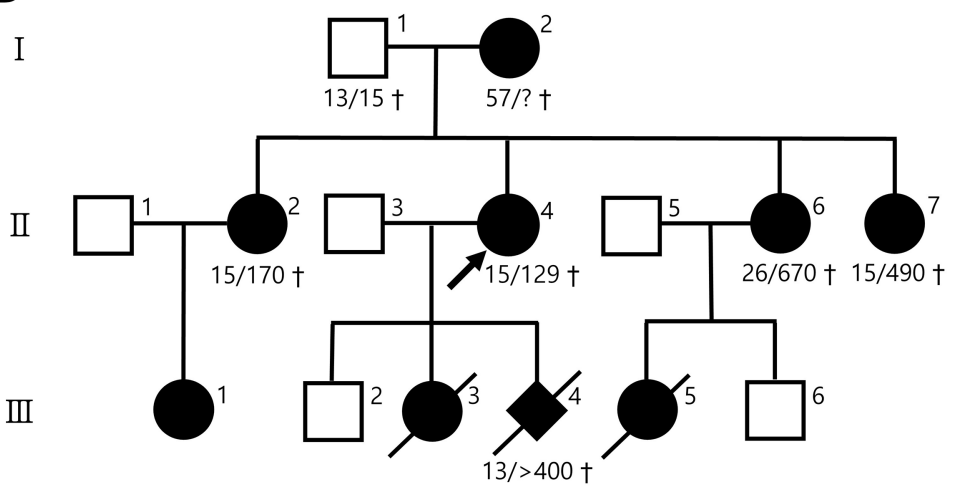

GW 32.0 GW 22.3 GW 32.0

Fig. 1. Pedigree pattern demonstrating an autosomal dominant myotonic dystrophy in 5 families. Arrow shows the proband of each case. (A) Case 1 , (B) Case 2, (C) Case 3, (D) Case 4.

GW, gestation weeks; ${ }^{\dagger}$ CTG repeats.

tubal factor and a previous history of fetal neural tube defects. She was pregnant with dichorionic diamniotic twins following cryopreserved embryo transfer. No specific abnormalities were noted on early ultrasound (US) exam during antenatal care in the first trimester; crown-rump lengths were 62 and $63 \mathrm{~mm}$ for the first and second fetuses, respectively, and nuchal translucency was within the normal range (1.5 and $1.6 \mathrm{~mm}$, respectively). During the second trimester, her pregnancy was complicated, with a preterm labor, bilateral borderline ventriculomegaly of the first fetus, relatively large amniotic volume, decreased movement of both fetuses, and maternal thrombocytopenia. At 30 weeks and 3 days of gestation, she underwent emergency cesarean section due to premature rupture of membranes. The first male twin baby was delivered weighing 1,460 $\mathrm{g}$ with an Apgar score of 1,2, and 4 at 1,5 and 10 minutes, respectively. The second male twin baby was delivered weighing 1,325 g, with an Apgar score of 3 and 5 at 1 and 5 minutes, respectively. At birth, the heart rate of the first baby was recovered after resuscitation. Both premature newborns appeared to be hypotonic with poor activity and developed respiratory discomfort, such that they required intubation for ventilator support in the neonatal intensive care unit (NICU). Severe hypotonia and persistent respiratory difficulties were observed in both newborns, III-1 and III-2 (Fig. 1A), both were determined by Southern blot to have one allele with more than 750 CTG repeats, and were diagnosed with CMD. The mother also had the complication of postpartum hemorrhage (PPH) due to uterine atony, with an estimated blood loss of 3,000 $\mathrm{mL}$ during the cesarean section, and needed modified B-lynch's sutures with massive transfusion. In addition, she was suspected of retinal detachment at 3 days postpartum, so that she was transferred to the tertiary center. Both infants III-1 and III-2 expired owing to respiratory failure at 3 and 4 months postnatal, respectively. After that, the mother (proband, II-2) (Fig. 1A) was confirmed as classic type DM1 with one allele with 130 CTG repeats.

\section{Case 2}

A 31-year old Korean pregnant woman, II-6 (Fig. 1B), gravida 
1 para 0 at 13 weeks and 5 days of gestation presented to our institution for antenatal care after pregnancy by intrauterine insemination. She had no previous medical history, but two consecutive babies (III-3 and III-4) of her older sisters, II-4 (Fig. 1B) were died immediately after birth with unknown cause. Ultrasonography showed no specific findings with normal amniotic fluid amount during the pregnancy. Her pregnancy was unremarkable except 2 admissions for tocolytic treatment due to preterm labor. A male newborn, III-5 (Fig. 1B), was delivered by cesarean section due to cephalopelvic disproportion at 38 weeks and 3 days of gestation. His birth weight of 3,685 g and length were appropriate for his gestational age, and Apgar scores at 1 and 5 minutes were 6 and 8, respectively. After birth, the newborn showed poor activity with hypotonic features and demonstrated respiratory insufficiency requiring intubation for ventilator support in the NICU. A complete physical examination and evaluation of hypotonia were performed for this newborn by pediatrician. Prior to pregnancy, the mother reportedly had handgrip myotonia, which caused her to take more time when writing by hand. Genetic testing of this newborn confirmed CMD, with one allele with more than 400 base pair repetitions, inherited from the mother, who was also confirmed to have DM1, with one allele with more than 400 base pair repetitions. This newborn showed developmental delay and an ophthalmological problem in an ongoing follow-up pediatric outpatient clinic until 5 years postnatally.

\section{Case 3}

A 33.5-year old woman, II-2 (Fig. 1C), gravida 2, para 0 at 13 weeks and 5 days of gestation was referred following natural pregnancy. Her pregnancy was complicated by a short cervix length, and she needed a McDonald cerclage at 15 weeks gestation. Amniotic volume has increased in the third trimester. She delivered a premature male newborn, III-2 (Fig. $1 \mathrm{C})$ at 36 weeks and 1 days due to premature rupture of the membranes. The body weight of the newborn was appropriate at 2,480 g, and the Apgar score was 6, 4, and 7 at 1,5 and 10 minutes, respectively. However, he developed respiratory discomfort complications at birth and required intubation for ventilator support in the NICU. An evaluation for hypotonia revealed that the newborn had CMD with 13/>1,080 base pair CTG repeats. In addition, brain US revealed leukomalacia. The mother (proband, II-2) was suspected to have DM1 because of her manifestation of handgrip myotonia, gait disturbance, and slender face. Genetic analysis using genomic DNA from the mother confirmed DM1 with >280 CTG repeats. Her sisters (II-
$3,4,5)$ were recommended for genetic counseling and testing, and all were confirmed as having DM1. A linkage analysis and molecular testing of family members revealed that the abnormal CTG repeats in the proband (II-2) came from her father, I-1 (Fig. 1C). After discharge, the affected baby III-2 (Fig. 1C) needed assistance to walk until 24 months and required rehabilitation training owing to developmental delay. The baby was lost to follow up at 2 years and 3 months.

\section{Case 4}

A 35.7-year old woman, II-4 (Fig. 1D), gravida 3, para 1 at 15 weeks of gestation presented because of a family history of CMD and a high risk of Down syndrome in prenatal screening. Her previous female baby, III-3, delivered at 32 weeks of gestation and expired at births, and had been confirmed as having CMD by genetic analysis (Fig. 1D). Her older sister's baby (III-1) had mental retardation and was undergoing rehabilitation treatment, and individual II-6's baby (III-5) had history of hypotonic features and neonatal death following preterm births at 32 weeks of gestation. Both II-6 and II-7 had difficulties walking. In her family, individuals II-2, II-4, II-6, and II-7 were each diagnosed with DM1, inherited from their mother, as shown by clinical examination and genetic analyses (Fig. 1D). In the proband, II-4 (arrow), a single mutant allele with more than 129 base pair CTG repeats was detected. In amniocentesis of the proband II-4, fetus (III-4) had 13/>400 base pair alleles CTG repeats, which is suspected to be congenital or classic type DM. In addition, abnormal fetal US findings, such as bilateral borderline ventriculomegaly, prominent nuchal fold thickness and bilateral superior vena cava were observed at 19 weeks of gestation. Following genetic counseling with the parents, the parents decided to terminate the pregnancy at 22 weeks of gestation. The termination of the pregnancy was complicated by $\mathrm{PPH}$, and the patient required transfusion. A planning pregnancy using preimplantation genetic diagnosis (PGD) was recommended for future pregnancies.

\section{Discussion}

This is the case series report on pregnancy outcomes and genetic counseling for family planning in DM patients in Korea. Taken together, the cases showed complications during either the pregnancy or postpartum period in DM1 women, and severe hypotonic features in CMD newborns at birth. Early diagnosis of DM1 in reproductive women without previously known family history is very difficult because it is a rare genetic disorder and 
patients can be asymptomatic or have only mild symptoms, such as myotonia before diagnosis. Even though, complications of DM1 during pregnancy or delivery were unspecific, including reduced fetal movements, increased amniotic fluid volume, preterm birth or PPH, asymptomatic pregnant women with DM1, or carriers having unstable CTG repeats or premutation alleles can have newborns with CMD by anticipation (i.e., the expansion of CTG repeats in the next generation). The clinical characteristics of the case series of CMD are listed in Table 1.

Most CMD patients have severe hypotonic features and expire due to severe respiratory insufficiency at birth, which accounts for the high rate of mortality in CMD patients [3]. In this study, three cases in which the mother (arrow) was the DM1 proband were not diagnosed until after delivery (Figs. 1A-C). DM type 2 shares most of the clinical features of DM1, except that there is no associated congenital presentation. All affected newborns received the amplified alleles from the mother with expanded CTG repeats. However, the family history of DM1 was unknown in three cases (Cases 1, 2, and 3) prior to birth, and all DM1 mothers (Cases 1-4) had a poor obstetric history, with preterm labor and incompetent cervix, respectively. There were two cases of fetal ventriculomegaly (Cases 1 and 3), two cases of increased amniotic volume (Cases 1 and 4), and one case of decreased fetal movements (Case 1). However, it is difficult to suspect CMD with only these antenatal findings, which can be seen even in normal fetuses. In previous studies, pregnant DM1 patients often have preterm labor, and fetuses with CMD manifest reduced fetal movements, polyhydramnios, talipes, and typical inverted V-shaped upper lip known as "carp mouth," which is a characteristic of severe facial weakness and causes a weak cry and the inability to suck [4-9]. Despite these unspecific antenatal signs, CMD fetuses can have severe symptoms at birth. In the affected offspring in the present cases (Cases 1-4), all newborns demonstrated hypotonic features with respiratory

Table 1. Clinical characteristics of cases of DM mother give births to congenital myotonic dystrophy

\begin{tabular}{|c|c|c|c|c|}
\hline Variable & Case 1 & Case 2 & Case 3 & Case 4 \\
\hline Known family history of DM1 & N & Suspected & N & Y \\
\hline Previous CMD births & N & N & N & Y \\
\hline Gravida/parity & $3 / 0$ & $1 / 0$ & $1 / 0$ & $3 / 1$ \\
\hline Method of pregnancy & C-ET & $|U|$ & Natural & Natural \\
\hline CTG repeats in affected grand parents & Unknown & Unknown & 59 (GF) & 57 (GM) \\
\hline CTG repeats in affected parents & $12 / 130$ & $13 />400$ & $5 />280$ & $15 / 129$ \\
\hline CTG repeats in affected offspring & $\begin{array}{l}\text { A: } 23 />750 \\
\text { B: } 13 />750\end{array}$ & $13 />400$ & $12 />1,080$ & $13 />400$ \\
\hline Sex of proband's offspring & Male/male & Male & Male & Male \\
\hline Types of DM in fetus & Congenital & Classic or congenital & Congenital & Classic or congenital \\
\hline GA at birth (wk) & 30.3 & 38.3 & 36.6 & 22.3 \\
\hline Birth weight (g) & $1,460 / 1,325$ & 3,685 & 2,480 & \\
\hline Apgar score at 1 minutes & $1 / 3$ & 6 & 6 & 0 \\
\hline Apgar score at 5 minutes & $2 / 5$ & 8 & 4 & 0 \\
\hline Postnatal intubation & Y/Y & Y & Y & \\
\hline NICU care & $Y / Y$ & Y & Y & \\
\hline Increased AFV & Y & N & Y & \\
\hline Decreased FM & Y & N & Y & \\
\hline Ventriculomegaly & Y & N & N & Y \\
\hline Obstetric problems & PTB, PPH & N & ॥OC, РТВ & PPH \\
\hline Maternal hand grip myotonia & Y & Y & Y & Y \\
\hline Maternal distal muscle weakness & N & Y & Y & Y \\
\hline Neonatal death & Y & N & N & \\
\hline Postnatal developmental delay & N & N & Y & \\
\hline Duration of postnatal follow-up (mo) & $3 / 4$ & 4 & 24 & \\
\hline
\end{tabular}

DM, myotonic dystrophy; DM1, myotonic dystrophy type 1; N, no; Y, yes; CMD, congenital myotonic dystrophy; C-ET, cryopreserved embyo transfer; IUI, intrauterine insemination; CTG, cystosine-thymine-guanine; GF, grandfather; GM, grandmother; GA, gestational age; NICU, neonatal intensive care unit; AFV, amniotic fluid volume; FM, fetal movements; PTB, preterm birth; PPH, postpartum hemorrhage; IIOC, incompetent cervix. 
insufficiency. Severity of respiratory insufficiency is the most critical factor for survival, and gestational weeks of delivery also seems to be important. In Case 1, a preterm twin CMD case born at 30 weeks of gestation died before 6 months. A surviving CMD newborn, though rare, can experience gradual improvement in motor function (i.e., they can swallow and independently ventilate). Almost all surviving CMD children are able to walk; however, cognitive and motor milestones are delayed and all CMD children develop learning difficulties and require special needs schooling [10-12]. In the literature, most cases were of inherited CTG repeats from the mother. Maternal inherited CMD has more severe hypotonic symptoms than those seen in patients with DM1 of paternal inheritance.

There were some difficulties in the prenatal diagnosis of CMD. First, the variable penetrance and expression of DM 1 make it a difficult condition to diagnose due to unspecific early symptoms. Anticipation, the expansion of CTG repeats in the next generation, can occur in asymptomatic women with premutation alleles. Second, somatic mosaicisms (i.e., the possibility of the presence of alleles having different size CTG repeats in the different tissues of the same individual) hampered prenatal diagnosis. Studies have shown variations of CTG repeat expansions in different tissues [13]. Therefore, based on the history and clinical symptoms of parents including kinships, diagnosis should be confirmed by molecular genetic testing, the gold standard for the diagnosis of DM1 or CMD. This is especially important for prenatal genetic diagnosis, for which the reliability and speed are crucial. In family planning in known affected women, especially infertile couples, we usually recommended in vitro fertilization and embryo transfer following PGD. However, PGD is technically difficult to perform. Furthermore, to succeed in having unaffected offspring, the affected or carrier parents should not share the same numbers of normal CTG repeats in the DM protein kinase allele. This requirement is necessary in order to exclude misinterpretation caused by overall amplification failure or allele dropout of an expanded allele. To avoid this mistake, a modified triplet-repeat polymerase chain reaction protocol has been recommended [14]. In our center, combined tests including both polymerase chain reaction (PCR) and Southern blot have been used for DM1 and CMD. PCR is a rapid prenatal test for the discovery of small CTG repeats (less than 100 repeats), and longer CTG repeats (more than 1,000 repeats) cannot be detect by PCR; hence Southern blotting using genomic DNA is used.

This report is expected to be helpful for understanding the obstetric outcomes and postnatal care of DM1. When counseling affected individuals or suspected DM1 families, physicians should inquire about family history (and organize a pedigree if needed), presence of DM1 associated systemic symptoms or signs, obstetrical history of female kinships, and, finally, recommend molecular genetic testing in suspected family members. If individuals without known family history have poor obstetric history, such as infertility, preterm labor, or sudden death of previous newborn at birth, obstetricians should carefully evaluate physical signs, such as myotonia in parents, and check the pedigree. Information regarding systemic effects, inheritance, and poor pregnancy outcomes on future offspring of these genetic disorders should be given to affected parents or partners. If individuals with a known family history of DM1 or CMD visited a clinic after pregnancy, or conceived fetuses are suspected to have CMD, a physician can consider amniocentesis, chorionic villus sampling, or cordocentesis for genetic testing. In addition, for couples with a family history of DM who are planning conception in advance, PGD can be recommended for especially subfertile couples. In conculsion, clinicians, especially obstetricians, should be aware of the potential for poor obstetric outcomes in women with DM and require attending neonatologists after birth due to severe postnatal respiratory insufficiency at birth. However, couples with a family history of DM1 should not be discouraged from conceiving due to fear of transmission, and the desires of the family should be respected as to whether or not they wish for the genetic study to be undertaken. Meticulous counseling for family planning should be offered to both probands and their family members, with information about diagnosis, obstetric outcomes, prognosis, and possible options in pregnancy.

\section{References}

1. Brook JD, McCurrach ME, Harley HG, Buckler AJ, Church D, Aburatani $\mathrm{H}$, et al. Molecular basis of myotonic dystrophy: expansion of a trinucleotide (CTG) repeat at the 3 ' end of a transcript encoding a protein kinase family member. Cell 1992;69:385.

2. Harris $S_{1}$ Moncrieff $C$, Johnson K. Myotonic dystrophy: will the real gene please step forward! Hum Mol Genet. 1996;5:1417-23.

3. Reardon W, Newcombe R, Fenton I, Sibert J, Harper PS. The natural history of congenital myotonic dystrophy: mortality and long term clinical aspects. Arch Dis Child 1993;68:177-81.

4. Sarnat HB, O'Connor T, Byrne PA. Clinical effects of myotonic dystrophy on pregnancy and the neonate. Arch Neurol 1976;33:45965.

5. Webb DA. The hazards of pregnancy in myotonic muscular dystrophy 
Mater Med Pol 1979;11:394-7.

6. Schild RL, Plath $H_{1}$ Hofstaetter $C$, Brenner R, Mann E, Mundegar RR, et al. Polyhydramnios: an association with congenital myotonic dystrophy. J Obstet Gynaecol 1998;18:484-5.

7. Zaki M, Boyd PA, Impey L, Roberts A, Chamberlain P. Congenital myotonic dystrophy: prenatal ultrasound findings and pregnancy outcome. Ultrasound Obstet Gynecol 2007;29:284-8.

8. Mashiach R, Rimon E, Achiron R. Tent-shaped mouth as a presenting symptom of congenital myotonic dystrophy. Ultrasound Obstet Gynecol 2002;20:312-3.

9. Levine AB, Eddleman KA, Chitkara U, Willner JP, Vosatka RJ, Berkowitz RL. Congenital myotonic dystrophy: an often unsuspected cause of severe polyhydramnios. Prenat Diagn 1991;11:111-5.

10. Spranger M, Spranger $S$, Tischendorf $M$, Meinck HM, Cremer M. Myotonic dystrophy. The role of large triplet repeat length in the development of mental retardation. Arch Neurol 1997;54:251-4.

11. Ashizawa T. Myotonic dystrophy as a brain disorder. Arch Neurol 1998;55:291-3.

12. Turner $C_{1}$ Hilton-Jones $D$. The myotonic dystrophies: diagnosis and management. J Neurol Neurosurg Psychiatry 2010;81:358-67.

13. Jansen $G$, Willems $P$, Coerwinkel $M$, Nillesen $W$, Smeets $H$, Vits $L$, et al. Gonosomal mosaicism in myotonic dystrophy patients: involvement of mitotic events in (CTG)n repeat variation and selection against extreme expansion in sperm. Am J Hum Genet 1994;54:575-85.

14. Kakourou G, Dhanjal S, Mamas T, Serhal P, Delhanty JD, SenGupta SB. Modification of the triplet repeat primed polymerase chain reaction method for detection of the CTG repeat expansion in myotonic dystrophy type 1: application in preimplantation genetic diagnosis. Fertil Steril 2010;94:1674-9. 